\title{
Evaluation of Collagen-Polyurethane-Chitosan Hydrogels for Lead Ions Removal from Water
}

\author{
Reyes-Ruiz Irene D', Cesar E. Castañeda-Calzoncit ${ }^{1}$, Jesús A. Claudio Rizo ${ }^{1 *}$, Tirso E. Flores-Guía', Denis A. \\ Cabrera-Munguía', Lucía F. Cano-Salazar' \& Juan J. Becerra-Rodriguez ${ }^{2}$ \\ ${ }^{1}$ Universidad Autónoma de Coahuila, Facultad de Ciencias Químicas, Ing J.Cardenas Valdez S/N, República, 25280 Saltillo, \\ Coahuila, México. E-mail: jclaudio@uadec.edu.m $x^{1 *}$ \\ ${ }^{2}$ Universidad Politécnica de Pénjamo, Ingeniería en Biotecnología, Carretera Irapuato, A La Piedad Km 44, 36900 El \\ Derramadero, Pénjamo, Guanajuato, México.
}

DOI: $10.46382 / M J B A S .2020 .4209$

Article Received: 27 February 2020

Article Accepted: 29 May 2020

Article Published: 30 June 2020

ABSTRACT

Collagen-polyurethane-chitosan hydrogels were synthesized by modifying the chemical structure of the crosslinking agent, with the aim to test which one plays a better role in removing of lead ions from water through adsorption process. In the first instance, two chemical crosslinkers based on aqueous polyurethane prepolymers (PPU) were used, where the type of aliphatic diisocyanate: hexamethylene diisocyanate $P(H D I)$ or isophoronadiisocyanate $P(I P D I)$ was varied. Hydrogels were subsequently designed using type I collagen $(C)$ and chitosan $(Q)$ varying the type of crosslinker: $C Q-P(H D I)$ and CQ-P(IPDI), respectively. Hydrogels were characterized by means of crosslinking index, infrared spectroscopy (FTIR), thermogravimetric behavior (TGA) and swelling/degradation kinetics. Finally, tests were performed to determine the removal rate of Pb (II) ions in model waters. The results indicate that $C Q-P(H D I)$ hydrogels have a higher degree of crosslinking, improving its resistance to the both thermal and hydrolytic degradation, and higher swelling capacity at acidic $\mathrm{pH}$; compared to those derived from $C Q-P(I P D I)$; however, these hydrogels do not show a decrement in the removal rate of Pb (II) ions from water, compared to the CQ hydrogel (without crosslinking), thus these innovative materials could be used as an alternative with potential use in the remediation of waters contaminated with lead ions.

Keywords: Hydrogels, Chemical crosslinking, Collagen, Chitosan, Polyurethane, Lead.

\section{Introduction}

Water is an elemental resource for the development and life and organisms. The contamination of water by harmful chemical agents has occurred frequently, and is a problem of the utmost importance, since it directly affects ecosystems and puts the health of humans at risk ${ }^{[1-2]}$. Metallic lead is widely used as a raw material in industrial processes, such as the mining and smelting industry, and their residues contaminate the aquifers ${ }^{[2]}$. In this sense, lead ions ( $\mathrm{Pb}$ (II), Pb (IV)) accumulate in the environment and endanger health, also damaging the intellectual development of children. Exposure to this ion is due to poor regulation in the disposal of contaminated industrial wastewater. Even in trace amounts (less than 50 ppm), the lead ions remain a highly contaminant ${ }^{[3-4]}$.

Due to this, different techniques have been used for the removal of lead ions from water, such as: chemical precipitation, ion exchange, membrane filtration, adsorption, coagulation, flocculation, among others ${ }^{[5-6]}$. Adsorption has been considered one of the most effective methods for removing pollutants from water, since it is usually inexpensive, and it does not generate waste problems [7-10]. The efficiency of the 
adsorption majority depends on the adsorbent material. Conventional adsorbent materials to remove heavy metal ions, such as: zeolite, clay, peat moss, and coal have exhibited a good response ${ }^{[9-10]}$.

However, it is necessary to develop novel strategies for the generation of novel adsorbent materials with potential application in wastewater remediation, representing easy applicability and high adsorption efficiency. Adsorbent materials in the hydrogel state have shown excellent capacity for adsorption of metal ions, having a high advantage over conventional adsorbents in that they do not require subsequent purification techniques for adsorbent separation ${ }^{[11-13]}$. However, it is necessary to modulate the mechanical properties and degradation of these matrices in the hydrogel state, in order to potentiate their use in both environmental and industrial strategies.

Hydrogels based on natural biopolymers such as collagen and chitosan have shown excellent adsorbent capacity for metal ions, however their application is limited by matrix degradation induced by conditions where these ions tend to prevail [14-16]. Therefore, it is ideal to develop strategies to control the degradation stability of these matrices under these conditions. Chemical crosslinking of collagen with polyurethane has been shown to be an effective method for developing hydrogels with controlled degradation; it also allows the coupling of external polymer chains to generate semi-interpenetrated systems in the hydrogel state ${ }^{[17]}$. With this in mind, this work proposes the evaluation of novel collagen-polyurethane-chitosan hydrogels with potential application for the removal of lead ions from water. The work contemplates studying the effect of the chemical structure of the polyurethane crosslinker on the removal capacity of these ions. For this, the crosslinking index, the chemical structure of the hydrogels evaluated by infrared spectroscopy (FTIR), thermogravimetric analysis (TGA) and swelling/degradation kinetics are also analyzed.

\section{Experimental Methodology}

\subsection{Synthesis of hydrogels based on collagen-polyurethane-chitosan}

Type I collagen from bovine tendon was extracted as previously reported ${ }^{[18]}$. Chitosan with a degree of deacetylation of $60 \%$ was obtained using the reported methodology ${ }^{[19]}$. Crosslinkers based on aqueous polyurethane dispersions were synthesized using polyethylene glycol (PEG) $(1000 \mathrm{~g} / \mathrm{mol})$, hexamethylenediisiocinate (P(HDI)) or isophoronadiisocyanate (P(IPDI)), as reported in the literature ${ }^{[20]}$. 
Semi-interpenetrated networks (semi-IPN) hydrogels were synthesized using the plate microemulsion method, for this: $1 \mathrm{ml}$ of a standardized collagen solution $(6 \mathrm{mg} / \mathrm{mL})$ was crosslinked using a 15 weight \% (wt.\%) ratio of crosslinking agent $\mathrm{P}$ (HDI) or $\mathrm{P}$ (IPDI), as the case may be. The crosslinking reaction was carried out at $\mathrm{pH} 7.0$ at $4^{\circ}$ $\mathrm{C}$ for $2 \mathrm{~h}$. Subsequently, once the viscosity of the systems increased, $15 \mathrm{wt} \%$ of chitosan was added. The formation of the semi-interpenetrated networks in the hydrogel state was carried out at $37^{\circ} \mathrm{C}$ for 18 . In Table 1 , the compositions and designations of the hydrogels prepared in this work are presented.

\begin{tabular}{|c|c|c|c|}
\hline $\begin{array}{c}\text { Bovine Type I } \\
\text { collagen (6 } \\
\mathbf{m g} / \mathbf{m L}) / \mathbf{m L}\end{array}$ & Chitosan/wt.\% & $\begin{array}{c}\text { Type of crosslinker } \\
\text { used in } \mathbf{1 5} \mathbf{w t . \%} \text { ratio }\end{array}$ & $\begin{array}{c}\text { Hydrogel } \\
\text { designation }\end{array}$ \\
\hline 1 & 15 & CQ \\
\hline 1 & 15 & P(HDI) & CQ-P(HDI) \\
\hline 1 & 15 & CQ-P(IPDI) \\
\hline
\end{tabular}

Table 1. Formulation of the hydrogels under study

\subsection{Characterization of hydrogels}

The determination of crosslinking index of hydrogels was monitored with the ninhydrin test. For it, $1 \mathrm{~mL}$ of ninhydrin solution ( $1 \mathrm{wt}$ \%, citrate buffer, $\mathrm{pH} 5.0$ ) was added to each hydrogel. Then, the hydrogels were heated to $90^{\circ} \mathrm{C}$ for $30 \mathrm{~min}$. The mixture was cooled down to room temperature and diluted with $3 \mathrm{~mL}$ of distilled water. The absorbance of the solution was determined at $567 \mathrm{~nm}$ employing a Lambda 35 Perkin-Elmer (UV-VIS) spectrophotometer. To assay the chemical structure of semi-IPN hydrogels, ATR-FTIR spectra with a Frontier, Perkin Elmer system, were recorded on fresh hydrogel composites at $16 \mathrm{~cm}^{-1}$ of resolution in a range from 3600 to $650 \mathrm{~cm}^{-1}$, using an average of 16 scans. The thermal behavior of the all dried hydrogels was evaluated by gravimetric thermal analysis (TGA), using a TGA-4000 Perkin Elmer thermoanalyzer.

The operating conditions used were: temperature range from 30 to $800^{\circ} \mathrm{C}$, heating rate of $20^{\circ} \mathrm{C} / \mathrm{min}$ using nitrogen as inert atmosphere. For the swelling behavior, the semi-IPN hydrogels were immersed in solutions at $\mathrm{pH} 5.0$ at $25^{\circ} \mathrm{C}$. Then, changes in 
the hydrogel mass over immersion time were assessed. The variation in mass (\%) of hydrogels was calculated according to the equation (1):

$$
\text { Mass variation }(\%)=\left[\left(m_{t}-m_{0}\right) / m_{0}\right] * 100 \quad \text { eq. }(1)
$$

Where $m_{0}$ is the initial mass of hydrogel and $m_{t}$ is the mass of sample at determined time.

\subsection{Evaluation of the removal rate of $\mathrm{Pb}(\mathrm{II})$ ions}

The evaluation of the $\mathrm{Pb}$ (II) ion removal rate of the semi-IPN hydrogels was evaluated using standard solution of $50 \mathrm{ppm}$ of said ion. For this, $5 \mathrm{~mL}$ of standard solution were added in $50 \mathrm{~mL}$ Eppendorf tubes, then a hydrogel (100 mg of adsorbent) was added. The system was incubated at $25^{\circ} \mathrm{C}$ for $24 \mathrm{~h}$ at $\mathrm{pH} 5.0$, then the hydrogel was removed and the spectrophotometric determination of residual $\mathrm{Pb}$ (II) in the solutions was carried out. The photometric determination was made at $580 \mathrm{~nm}$ following the indications recommended by the manufacturer (Merck (109717)). The removal rate (\%) was calculated by the ratio among the residual and the initial $\mathrm{Pb}$ (II) concentrations in the solutions.

\subsection{Data analysis}

The mean and standard deviation (SD) are presented for each data set. Data sets were compared using analysis of variance (ANOVA). The difference of the means was checked with a Fisher Minimum Significant Difference test and was considered statistically significant at level $p<0.05$.

\section{Results and Discussion}

Pollution in water by $\mathrm{Pb}$ (II) ions is a matter of utmost importance because it puts the health of living beings at risk, and damages the ecosystems. In the present work, hydrogels were synthesized from semi-interpenetrated networks based on collagen-polyurethane-chitosan, with the aim of testing their capacity for removing the $\mathrm{Pb}$ (II) ions in water; providing new adsorbent materials that help with this environmental problem. The hydrogels were efficiently synthesized by the microemulsion method, and the main aim was to study the effect of the degree of crosslinking of the hydrogel, modifying the chemical structure of the crosslinking polyurethane $\mathrm{P}(\mathrm{HDI})$ or $\mathrm{P}(\mathrm{IPDI})$, on its physicochemical properties such as chemical 
structure, thermal degradation, swelling/degradation and removal rate of such metal ions.

The degree of crosslinking of the hydrogels was determined by the ninhydrin test. In Figure 1, the determined crosslinking indices are: $18 \pm 5 \%$ and $26 \pm 3 \%$ for CQ-P(IPDI) and $C Q-P(H D I)$, respectively. It can also be seen that there is a statistically significant difference in the crosslinking index of CQ-P(IPDI) hydrogel with respect to CQ-P(HDI). The isocyanate groups present in the hydrocarbon structure of HDI show greater reactivity for the formation of urea bonds with primary amino groups of collagen and chitosan, while the isocyanate groups present in IPDI, a cyclic structure, limits the crosslinking reactivity.

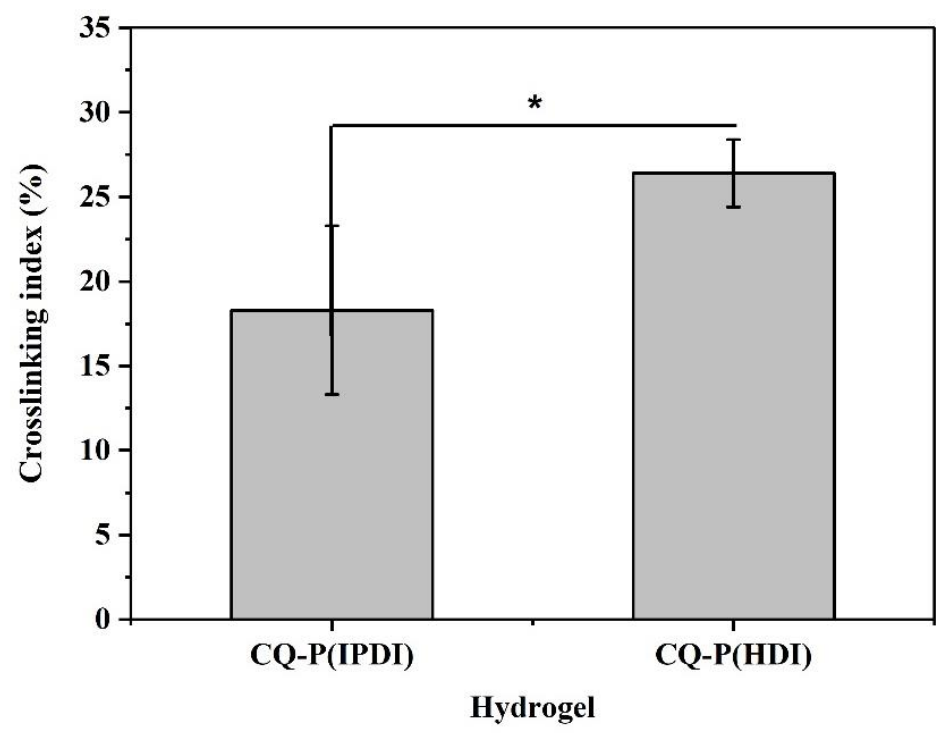

Figure 1. Determination of the crosslinking index of hydrogels of semi-interpenetrated networks based on collagen-polyurethane-chitosan

The crosslinking index values indicate that the 3D matrices in the hydrogel state formed by collagen-polyurethane-chitosan show higher semi-interpenetration when the crosslinking agent $\mathrm{P}(\mathrm{HDI})$ is used. Steric hindrance effects associated with the chemical structure of the isocyanate groups used show an important effect in the crosslinking of these biopolymeric chains. The linear aliphatic structure of HDI generates less steric hindrance than the aliphatic cyclic structure of IPDI, therefore the semi-IPN hydrogel based on $\mathrm{P}(\mathrm{HDI})$ considerably improves the physicochemical crosslinking $[17,20]$.

The modification of the chemical structure of the hydrogels was evaluated by FTIR. Figure 2 shows the FTIR spectra of the three hydrogels; the signal corresponding to the 
$\mathrm{N}-\mathrm{H}$ and $\mathrm{O}-\mathrm{H}$ bonds present in the structures of collagen, chitosan and polyurethane are displayed at 3500-3000 $\mathrm{cm}^{-1}$. The bands corresponding to the $\mathrm{C}-\mathrm{H}$ bonds of hydrocarbon chains are approximately in the region that comprises $2900-2700 \mathrm{~cm}^{-1}$; an increment in the intensity of these both signals can be seen for the Col-QP (HDI) and Col- QP (IPDI) semi-IPN hydrogels, evidencing so the presence of the polyurethane crosslinking agent.

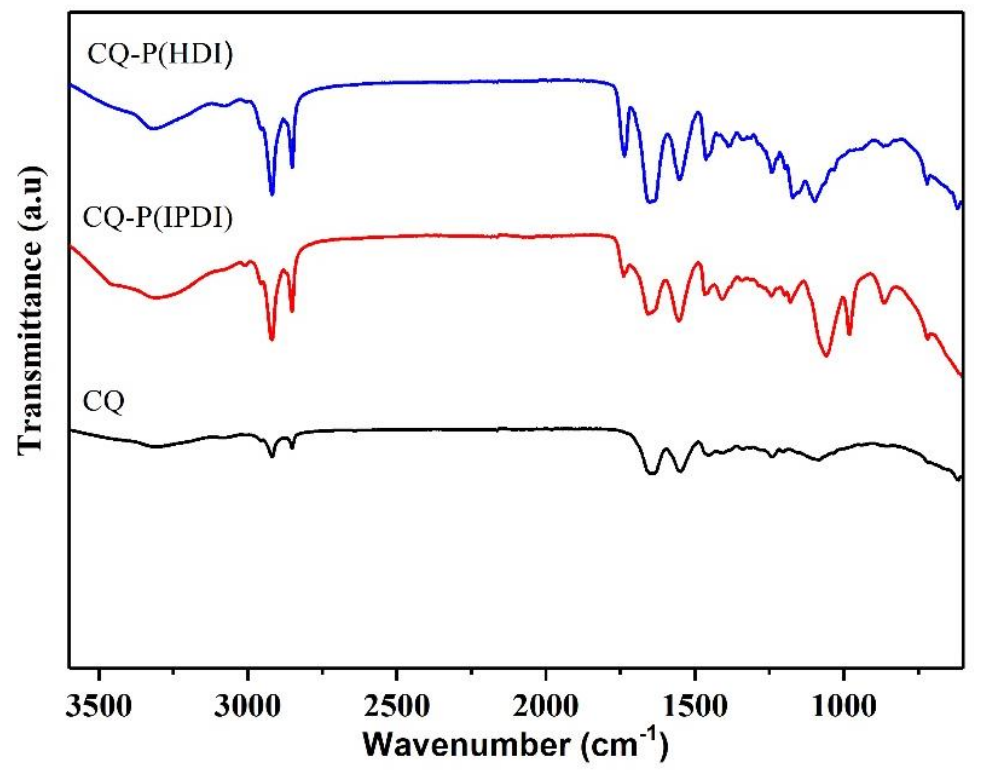

Figure 2. Evaluation of the chemical structure by FTIR

At $1700 \mathrm{~cm}^{-1}$ can observe the band corresponding to the urea crosslinking bond, which demonstrates the chemical crosslinking between the primary amino groups of the biopolymers with the isocyanate groups of the polyurethane, therefore this band only is present in CQ-P(HDI) and Col-QP(IPDI) hydrogels. This bond is generated within the semi-interpenetrated networks of the biopolymers with the polyurethane crosslinking agent. It is interesting to note that the intensity of this signal is higher in the CQ-P(HDI) hydrogel, indicating that there is a higher degree of crosslinking in this matrix, as indicated by the results of the ninhydrin test.

The bands at $1550 \mathrm{~cm}^{-1}$ and $1650 \mathrm{~cm}^{-1}$ corresponding to the amide I and amide II vibration modes of collagen, respectively, are also observed; displacements of these bands to lower wavenumbers also evidencing the chemical crosslinking process and the generation of a semi-IPN system.

The $1400-1000 \mathrm{~cm}^{-1}$ region displays the signals corresponding to the triple helical conformation of collagen. The modification in the intensity of the signals of this region 
also involves the fingerprint of the semi-IPN network based on collagen-chitosan, by adding the crosslinking agents $\mathrm{P}$ (HDI) or P(IPDI), this because the polyurethane chains can cover the semi-interpenetrated network of biopolymers; increasing the intensity of the $\mathrm{C}-\mathrm{O}$ bonds present around $1100 \mathrm{~cm}^{-1}$, which is dependent on the degree of crosslinking of the polymeric matrix, and therefore on the intermolecular interactions regulated by steric hindrance ${ }^{[20]}$.

Thermogravimetric analyzes were performed in order to understand the effect of crosslinking and the formation of semi-IPN networks on hydrogels based on collagen-polyurethane-chitosan. In figure 3, the TGA thermograms of the hydrogels evaluated are shown. Three regions of mass loss are visualized: i) the dehydration and elimination of volatile compounds in a temperature range among 50-120 ${ }^{\circ} \mathrm{C}$, ii) the thermal degradation of the polymeric components among 200-450 ${ }^{\circ} \mathrm{C}$ and iii) the generation of ash inorganic due to oxidation effects among 500-800 ${ }^{\circ} \mathrm{C}$.

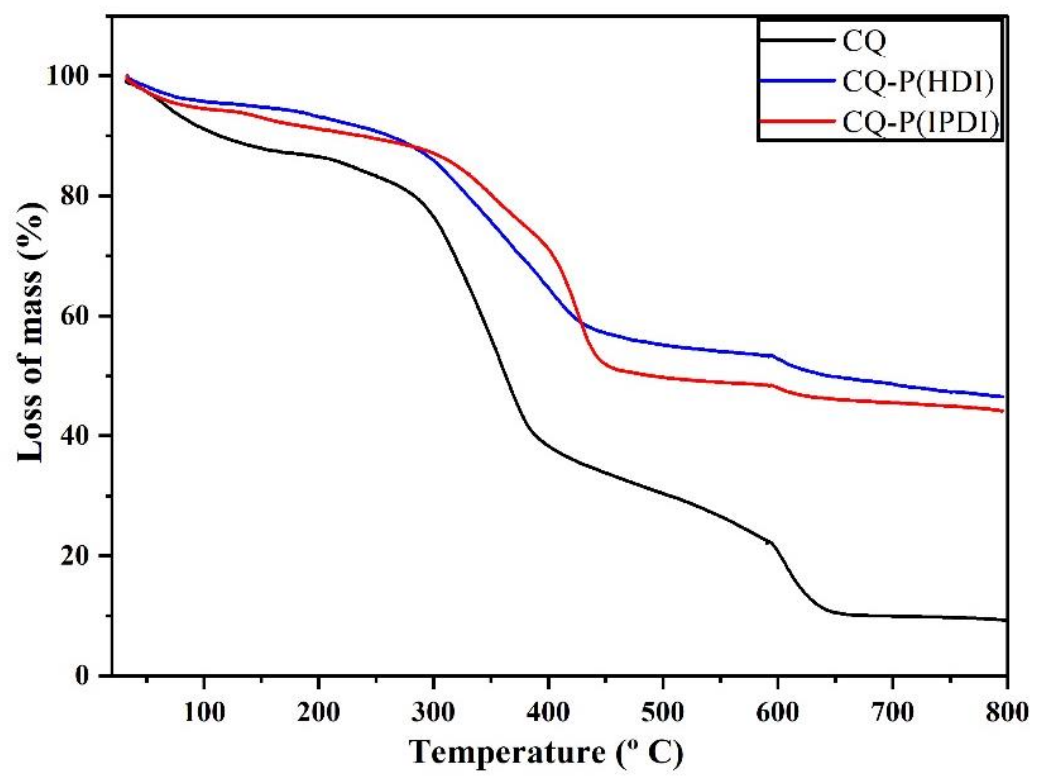

Figure 3. Evaluation of the thermal degradation of hydrogels by TGA

In the region that involves thermal degradation, losses of mass: $66 \pm 4 \%, 38 \pm 3 \%$ and $31 \pm 4 \%$ are obtained for CQ, CQ-P(IPDI) and CQ-P(HDI), respectively. A lowest mass loss is obtained for CQ-P(HDI) hydrogel, indicating that $\mathrm{P}(\mathrm{HDI})$ crosslinking agent improves the thermal degradation of collagen-quitosan matrix. The crosslinking urea bonds as well as weak intermolecular associations produced, such as hydrogen bridges and Vander Waals interactions, among the polymeric chains of collagen and chitosan with this crosslinking agent, are responsible for this improvement in thermal 
degradation. The resistance to thermal degradation has to be controlled in materials for applications in removal of heavy metal ions; since the increase in temperature can cause desorption of the trapped ions for the reuse of the adsorbent material ${ }^{[6-10]}$. In this sense, the uncrosslinked hydrogel based on collagen-chitosan shows high susceptibility to thermal degradation, which would limit its use as an adsorbent material in both industrial and environmental applications; while polyurethane crosslinked hydrogels provide thermal resistance improving their applicability as possible adsorbent materials.

The swelling behavior of hydrogels also represents an important parameter to control the removal capacity of heavy metal ions ${ }^{[12-15]}$. This behavior depends on the $\mathrm{pH}$, temperature and ionic strength ${ }^{[16-18]}$. In this sense, contaminating metal ions subsist in waste waters that have slightly acidic $\mathrm{pH}$, so it is important that the matrices in the hydrogel state show regulation of their swelling capacity at acidic $\mathrm{pH}$. The swelling behavior of the hydrogels was evaluated at $\mathrm{pH} \mathrm{5.0,} \mathrm{the} \mathrm{results} \mathrm{are} \mathrm{shown} \mathrm{in} \mathrm{figure} 4$.

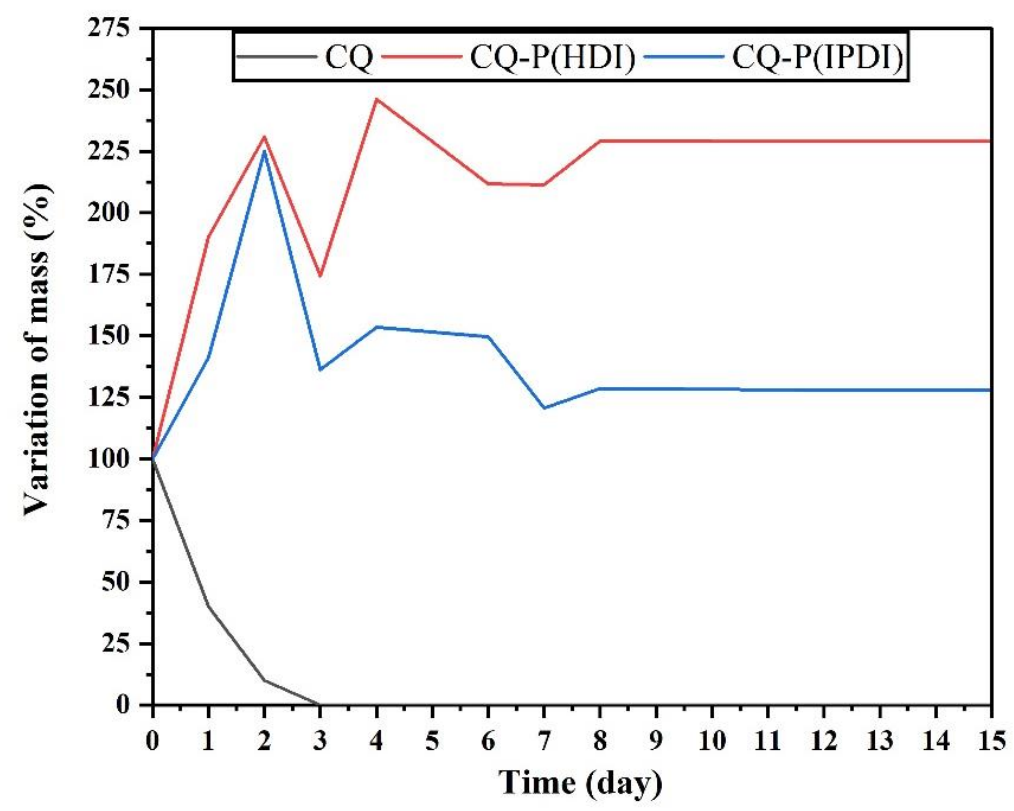

Figure 4. Swelling/degradation behavior of hydrogels at $\mathrm{pH} 5.0$

The results indicate that the CQ uncrosslinked hydrogel is completely degraded after 3 days of incubation at $\mathrm{pH}$ 5.0. Polyurethane crosslinked hydrogels show resistance to acid degradation; and they present swelling capacity. The matrix based on CQ-P(HDI) shows a swelling capacity of $223 \pm 18 \%$, while CQ-P(IPDI) exhibits a swelling of $124 \pm$ $12 \%$, after 8 days of incubation at $\mathrm{pH} 5.0$. Significantly statistical differences are found when comparing the swelling capacities of CQ-P(HDI) with respect to CQ-P(IPDI). The 
aliphatic regions formed by the cyclic structures of the P(IPDI) show less capacity to retain water molecules within the semi-IPN matrix, significantly decreasing their swelling capacity in acidic conditions. On the other hand, the linear regions of the $\mathrm{P}(\mathrm{HDI})$ crosslinker generate a matrix that does not have zones of steric impediment for the absorption of water molecules, notably improving its swelling behavior. According to these results, it also is evident that the CQ-based hydrogel would represent poor behavior as a metal ion adsorbent material, since this matrix would degrade as the process of adsorption of the contaminant is carried out, preventing its recovery and its subsequent reuse; while hydrogels crosslinked with $\mathrm{P}(\mathrm{HDI})$ or $\mathrm{P}$ (IPDI) could be recovered from the medium without significant loss mass due to hydrolytic degradation events.

Finally, the effect of the chemical structure of the polyurethane crosslinker of these collagen-chitosan semi-IPN matrices on their $\mathrm{Pb}$ (II) removal rates was evaluated. Figure 5 shows the removal rate of $\mathrm{Pb}$ (II) ions at $\mathrm{pH} 5.0$ during $24 \mathrm{~h}$. The removal rate obtained are: $99 \pm 8 \%, 99 \pm 9 \%$ and $96 \pm 8 \%$, for CQ, CQ-P(IPDI) and CQ-P(HDI) hydrogels, respectively.

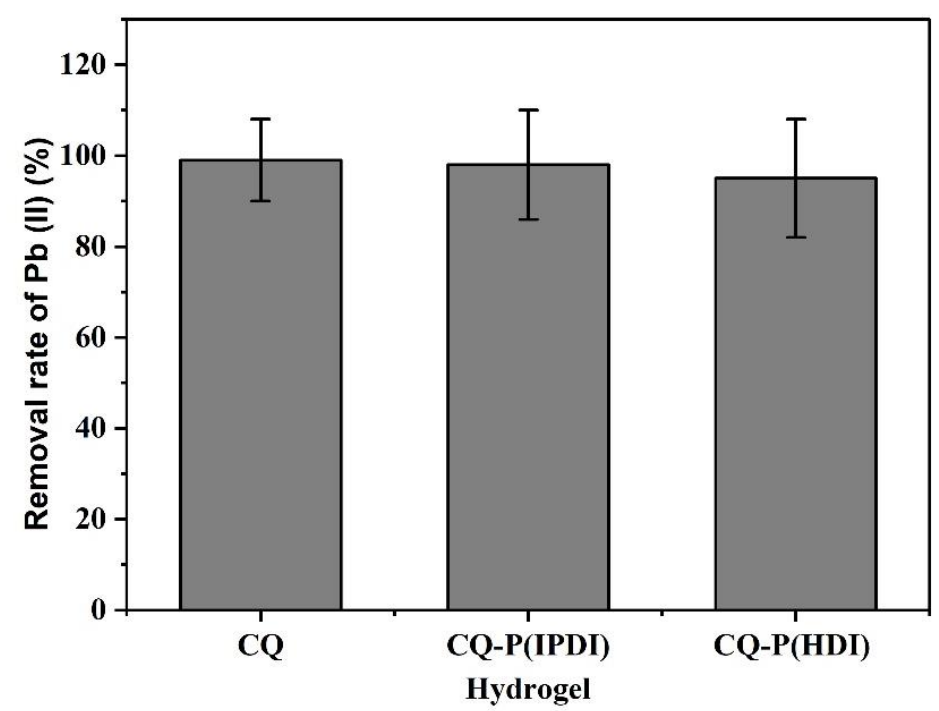

Figure 5. Evaluation of the removal rate of $\mathrm{Pb}$ (II) ions of studied materials

Statistically significant differences were not found between the removal rate results for the hydrogels studied. All hydrogels have excellent $\mathrm{Pb}$ (II) ion removal capacity, which is associated with their high content of nucleophilic groups such as $-\mathrm{NH},-\mathrm{OH}$, carboxylate and proline, located in their chemical structure. These groups are associated with chelation events and/or electrostatic attractions with the $\mathrm{Pb}$ (II) ions, 
favoring a high removal capacity of such ions [14-16]. The chemical structure of polyurethane does not limit the high adsorptive capacity inherent in collagen and chitosan biopolymers; however, it does give the semi-IPN matrix improved properties with respect to the $\mathrm{CQ}$ hydrogel for a better performance as a $\mathrm{Pb}$ (II) ion adsorbent in applications that require hydrogels with both regulated thermal and hydrolytic degradation. From the physicochemical point of view, this work provides important evidence in the development of novel adsorbent materials in the hydrogel state with improved properties for the $\mathrm{Pb}$ (II) adsorption process; therefore, studying both the thermodynamic and kinetic aspects of the adsorption process could represent a promising area of research that will allow us to understand the industrial and/or environmental applicability of these innovative matrices.

\section{Conclusion}

The generation of new adsorbent materials of $\mathrm{Pb}$ (II) ions in the hydrogel state is a promising area of research, due to the contamination of this ion in global aquifers. In this work, novel hydrogels based on semi-IPN collagen-polyurethane-chitosan networks were designed. The chemical structure of the polyurethane crosslinker was modified and its performance in removing $\mathrm{Pb}$ (II) ions was evaluated. The results show that CQ-P(HDI) hydrogels have a higher degree of crosslinking, improving its both resistance to the thermal and hydrolytic degradation, and higher swelling capacity at acidic $\mathrm{pH}$; compared to those derived from CQ-P(IPDI); however, these hydrogels do not show a decrement in the removal rate of $\mathrm{Pb}$ (II) ions from water compared to the $\mathrm{CQ}$ hydrogel (without crosslinking); therefore these materials could be potential candidates for the remediation of water contaminated by these ions, with excellent performance in acidic conditions or under high temperatures that do not limit the functionality of the adsorbent material.

\section{References}

[1] Nagajyoti P C, Lee K D, Sreekanth T V M. Heavy metals, occurrence and toxicity for plants: a review. Environmental Chemistry Letters, 2010, 8(3): 199-216.

[2] Rabin R. The lead industry and lead water pipes "A modest campaign". American Journal of Public Health, 2008, 98: 1584-1592.

[3] Landrigan P J. Lead and the heart: an ancient metal's contribution to modern disease, The Lancet Public Heath, 2018, 3: e156-e157. 
[4] Abadin H G, Hibbs B F, Pohl H R. Breast-feeding exposure of infants to cadmium, lead, and mercury: A public health viewpoint. Toxicology and Industrial Health, 1997, 15(4): $1-24$.

[5] Zhang W, Yang J, Wu X, Hu Y, Yu W, Wang J, Dong J, Li M, Liang S, Hu J, Kumar R V. A critical review on secondary lead recycling technology and its prospect. Renewable and Sustainable Energy Reviews, 2016, 61: 108-122.

[6] Sarma G K, Gupta S S, Bhattacharyya K G. Nanomaterials as versatile adsorbents for heavy metal ions in water: a review. Environmental Science and Pollution Research, 2019, 26: 6245-6278.

[7] Afroze S, Sen T K. A review on heavy metal ions and dye adsorption from water by agricultural solid waste adsorbents. Water Air Soil Pollut, 2018, 229: 225.

[8] Abdolali A, Guo W, Ngo H, Chen S, Nguyen N, Tung K. Typical lignocellulosic wastes and by-products for biosorption process in water and wastewater treatment: a critical review. Bioresource Technology, 2014, 160: 57-66.

[9] Qin Z, Liu F, Lan S, Li W, Yin H, Zheng L, Zhang Q. Effect of Y-manganite particle size on $\mathrm{Zn}^{2+}$ coordination environment during adsorption and desorption. Applied Clay Science, 2019, 168: 68-76.

[10] Ihsanullah A A, Al-Amer A M, Laoui T, Al-Marri M J, Nasser M S, Khraisheh M, Atieh M A. Heavy metal removal from aqueous solution by advanced carbon nanotubes: Critical review of adsorption applications. Separation and Purification Technology, 2016, 157: 141-161.

[11] Overstreet D J, Dutta D, Stabenfeldt S E, Vernon B L. Injectable hydrogels. Journal of Polymer Science Part B: Polymer Physics, 2012, 50(13): 881-903.

[12] Sinha V, Chakma S. Advances in the preparation of hydrogel for wastewater treatment: A concise review. Journal of Environmental Chemical Engineering, 2019, 7(5): 103295.

[13] Mahinroosta M, Farsangi Z J, Allahverdi A, Shakoori Z. Hydrogels as intelligent materials: A brief review of synthesis, properties and applications. Materials Today Chemistry, 2018, 8: 42-55.

[14] Vieira R M, Vilela P B, Becegato V A, Paulino A T. Chitosan-based hydrogel and chitosan/acid-activated montmorillonite composite hydrogel for the adsorption and 
removal of $\mathrm{Pb}^{+2}$ and $\mathrm{Ni}^{+2}$ ions accommodated in aqueous solutions. Journal of Environmental Chemical Engineering, 2018, 6(2): 2713-2723.

[15] Wang J, Wei L, Ma Y, Li K, Li M, Yu Y, Qiu H. Collagen/cellulose hydrogel beads reconstituted from ionic liquid solution for $\mathrm{Cu}(\mathrm{II})$ adsorption. Carbohydrate Polymers, 2013, 98(1): 736-743.

[16] Singha N. R, Roy C, Mahapatra M, Dutta A, Deb Roy J S, Mitra M, Chattopadhyay P K. Scalable synthesis of collagenic-waste and natural rubber-based biocomposite for removal of $\mathrm{Hg}(\mathrm{II})$ and dyes: Approach for cost-friendly waste management. ACS Omega, 2019, 4(1): 421-436.

[17] Claudio-Rizo J A, Mendoza-Novelo B, Delgado J, Castellano L E, Mata-Mata J L. A new method for the preparation of biomedical hydrogels comprised of extracellular matrix and oligourethanes. Biomedical Materials (Bristol), 2016, 11(3): 035016.

[18] Claudio-Rizo J A, Rangel-Argote M, Castellano L E, Delgado J, Mata-Mata J L, Mendoza-Novelo $B$. Influence of residual composition on the structure and properties of extracellular matrix derived hydrogels. Materials Science and Engineering: C, 2017, 79: 793-801.

[19] Hossain M S, Iqbal A. Production and characterization of chitosan from shrimp waste. Journal of the Bangladesh Agricultural University, 2014, 12(1): 153-160.

[20] Claudio-Rizo J A, Rangel-Argote M, Muñoz-González P U, Castellano L E, Delgado J, Gonzalez-García G, Mata-Mata J L, Mendoza-Novelo B. Improved properties of composite collagen hydrogels: protected oligourethanes and silica particles as modulators. Journal of Materials Chemistry B, 2016, 4(40): 6497-6509. 